\section{Japanese panel proposes extension of cancer research}

Tokyo. One of the most important sources of funds for cancer research and biological research in Japan seems likely to keep flowing for another decade. Earlier this month, a government working group of scientists with the backing of the Ministry of Health and

Nakasone. The joint funding by MHW, MESC and STA is unusual for Japan, where government ministries and agencies tend jealously to guard their turf. By March 1994 the three agencies will have spent more than $¥ 100$ billion (US\$900 million).

About half of the budget has been spent by STA on a heavy-particle accelerator for cancer treatment. The programme has also

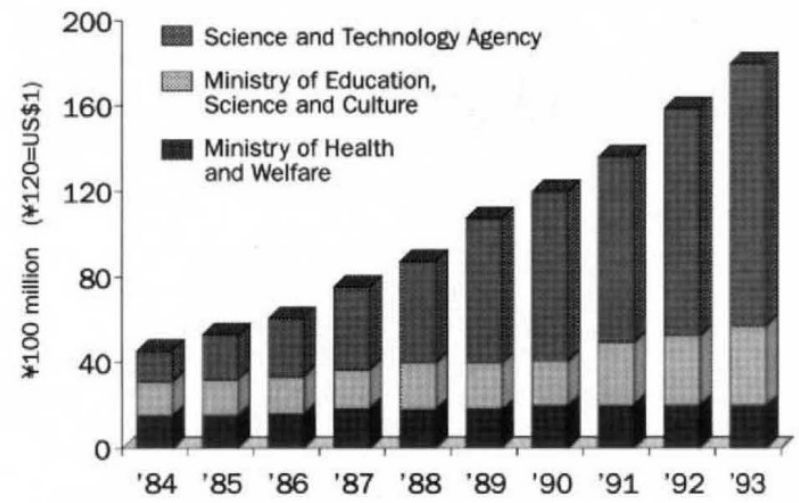

Welfare (MHW), the Ministry of Education, Science and Culture (MESC) and the Science and Technology Agency (STA) recommended the extension for another ten years of the Comprehensive Ten-Year Strategy for Cancer Control, with a slight shift towards more practical applications.

The cancer programme was launched in 1984 by then Prime Minister Yasuhiro are supported by MESC. provided a significant boost in research funds not only for cancer researchers working for MHW but also for molecular biologists, cell biologists, virologists and immunologists in Japan's universities, who

The working group, headed by Masaaki Terada, director of the National Cancer Centre Research Institute in Tokyo, recommends that the next phase should focus on seven topics (see table). New among them are research into cancer prevention and ways to improve the quality of life of cancer patients.

In line with the wishes of biologists working under the education ministry, the topics also include cell biology, immunology, virology and molecular research. Tadatsugu Taniguchi of Osaka University's

\section{Stretching the truth on cancer}

Tokyo. The Japanese Science and Technology Agency and the ministries of Health and Welfare and Education, Science and Culture have gone too far in extolling the achievements of their ten-year anti-cancer project.

A 12-page colour pamphlet issued by the three government organizations describes for the public an impressive list of accomplishments. Among them are the discovery that the HTLV-1 virus causes adult T-cell leukaemia (ATL) and that the hepatitus- $C$ virus is the cause of many cases of liver cancer. The pamphlet also includes an illustration of the drug Camptothecin under the heading "new anti-cancer drug treatments developed".

The problem is that the claims are not true. The link between HTLV-1 and ATL was established by Japanese and US researchers before the start of the project. The hepatitus-C virus was first isolated and characterized by Chiron, a US company, and Camptothecin was independently developed by a Japanese company, Yakult.

Masaki Mugitani, deputy director of the disease control division of the Ministry of Health and Welfare, says that the exaggerations are a result of writing for a general audience. A more technical report by and for scientists, he says, explains that the project helped to elucidate the mechanism by which HTLV-1 causes cancer and the route of infection and that it led to the discovery and characterization of the Japanese strain of hepatitus- $C$ virus.

The pamphlet is an embarrassment, says Masaaki Terada, director of the National Cancer Center Research Institute and leader of the working group that reviewed the project. It will be withdrawn and reissued after revision.

\section{An agenda for cancer research}

- Molecular mechanisms, including:

- oncogenes, tumour suppressor genes - radiation-induced cancer

Metastasis/invasion and characterization - mechanism and control

- abnormalities in expression, cell death

- Predisposition and immunology

- familial incidence

- Prevention, including:

- risk factors, epidemiology

- education

- New diagnostics

- imaging. early diagnosis

- New treatments

- gene therapy, drug tolerance

- heavy-particle beam

- Quality of life

- after-care, reducing pain

- economic factors

Institute for Molecular and Cellular Biology, an important figure in the present programme, says that he expects the programme to "continue to feed Japanese biology in general" because of its contribution to better understanding of the disease.

On the other hand, Yusuke Nakamura, chief of the division of biochemistry in the Cancer Institute, Tokyo, whose group of 40 researchers is expected to play an important role in the development of new diagnostics, says that his chief motivation is to "save patients' lives". He hopes to develop DNA diagnostics both for early diagnosis of cancer and to assess the degree of malignancy of tumours so that unnecessary surgery can be avoided.

The working group also called for research on gene therapy. "Many clinicians are interested", says Terada, but few Japanese scientists have research experience in gene therapy. The Japanese government has only recently initiated a process to develop guidelines and approval processes for such research, which for the foreseeable future will be confined to animals. The present ten-year programme also led to the establishment of the first postdoctoral positions supported by the Ministry of Health and Welfare, and it also funds a small exchange programme between Japanese and foreign scientists.

Although the programme's budget has yet to be determined, the working group hopes that research funds will be increased by about 50 per cent. Its report will undergo minor rewriting by its parent committee before approval is sought from the cabinet in time for the three agencies to submit their budget requests at the end of August.

David Swinbanks 